\title{
CONDUCTA INTENCIONAL EN NIÑOS CON TRASTORNOS POR DÉFICIT DE ATENCIÓN CON HIPERACTIVIDAD: RESULTADOS PRELIMINARES
}

\section{Volition In Attention Deficit Hyperactivity Disorder In Childhood: Preliminar Results}

\author{
Dulce María Romero Ayusoi, \\ Gary Kielhofner ii \\ Úrsula Arroyo Martínez iii
}

\section{RESUMEN}

La mayoría de los modelos teóricos que explican el trastorno por déficit de atención con hiperactividad coinciden en señalar la presencia de un déficit motivacional. Sin embargo, son pocos los estudios que se centran sobre la conducta intencional o volición en estos niños. El Modelo de la Ocupación Humana entiende que la volición es un elemento clave en la ocupación humana. El objetivo de este estudio es conocer cómo es la conducta intencional de los niños con trastorno por déficit de atención con hiperactividad para poder establecer objetivos y programas de tratamiento más eficaces con esta población. Método. La muestra está formada por 21 niños con edades comprendidas entre 7 y 11 años y todos ellos fueron evaluados a través del Pediatric Volitional Questionaire (PVQ) en dos ocupaciones: actividades de la vida diaria y actividades escolares. Resultados. Los resultados indican que la conducta intencional es diferente en las actividades de la vida diaria y actividades escolares, mostrando una mayor complejidad en las actividades escolares. La conducta intencional es inferior en los niños del subtipo combinado que el grupo control e inatento. Conclusiones. Estos resultados podrían indicar un menor desarrollo del sentido de capacidad o competencia en los niños con el trastorno por déficit de atención con hiperactividad. Los

\footnotetext{
'Terapeuta Ocupacional. Psicóloga. Dra. Psicología. Profesora Contratada Doctora, Subdirectora del Centro de Estudios Universitarios de Talavera de la Reina, Universidad de Castilla-La Mancha. Avda. Real Fábrica de Sedas, s/n. 45600 Talavera de la Reina (Toledo). España.+34 925721010 Ext. 5624. Fax: +34 9257210 11. Email: Dulce.Romero@uclm.es ii Dr PH, OTR/L, FAOTA Professor Wade/Meyer Chair, Department of Occupational Therapy. College of Applied Health Sciences. University of Illinois at Chicago. (312) 996-4973. Fax, (312) 413-0256. Email, kielhfnr@uic.edu
}

iii Terapeuta Ocupacional. Universidad de Castilla-La Mancha. Centro de Estudios Universitarios de Talavera de la Reina, Universidad de Castilla-La Mancha. Avda. Real Fábrica de Sedas, s/n. 45600 Talavera de la Reina (Toledo). España. +34 925721010 Ext. 5624. Fax: +34 925721011. 
tratamientos deberían fomentar el desarrollo de un sentido positivo de competencia y de identidad ocupacional

\begin{abstract}
Most of the theoretical models that explain Attention Deficit Hyperactivity Disorder suggest the presence of a motivational deficit. However, few studies that focuses on the intentional or volition in these children. The Model of Human Occupation understands that volition is a key element in the human occupation. The aim of this study is how the volition of children with Attention Deficit Hyperactivity Disorder in order to establish goals and treatment programs more effective with this population. Method. Twenty one children, between seven and eleven years old participated and were assessed through the Pediatric Volitional Questionnaire (PVQ) in two occupations: activities of daily living and school activities. Results. The results indicate that the volition is different in the activities of daily living and school activities, showing more complex school activities. The volition is lower in children in the combined subtype that the control group and inattentive subtype. Conclusions. These results could indicate a less developed sense of capacity or competence in children with attention deficit hyperactivity disorder. The future treatment should encourage the development of a positive sense of occupational identity and occupational competence.
\end{abstract}

Palabras Clave: volición, conducta intencional, trastornos de atención con hiperactividad, motivación, evaluación, ocupación, actividades de la vida diaria, actividades escolares

Key words: volition, attention deficit disorder with hyperactivity, motivation, assessment, occupation, activities of daily living, scholar activities.

\title{
INTRODUCCIÓN
}

¿Por qué es importante estudiar la conducta intencional de los niños con Trastornos por déficit de Atención (en adelante TDAH? ¿Qué importancia tienen las actividades de la vida diaria? ¿Qué puede aportar la terapia ocupacional al conocimiento de los trastornos por déficit de atención e hiperactividad? Estos tres interrogantes han sido las principales directrices del estudio que presentamos.

De acuerdo con $\mathrm{Chu}^{1}$, consideramos que "es importante apreciar que bajo las condiciones de laboratorio, los niños con TDAH no presentan ciertos déficit de atención sostenida. Pueden atender bien a tareas con frecuente tasa de refuerzo intrínseco" (Chu, 2003, pp.220). "La hiperactividad es más evidente durante actividades establecidas, actividades sentado en clase, durante la hora de comer o durante largos viajes y en actividades que no son cognitivamente cambiantes como el juego libre con otros”(1). 
Jensen et al. ${ }^{2}$ ven el TDAH como un trastorno de adaptación, de tal modo que el niño con TDAH puede funcionar diferencialmente en distintos entornos. Este autor entiende que los entornos son los contextos en los que el niño se involucra en diferentes actividades. Incluyen distintos factores físicos, sociales y culturales. En la figura 1 se ilustra la conceptualización de los trastornos por déficit de atención con hiperactividad en la infancia a partir de Jensen et al. (2).

Recientemente Tripp y Wickens ${ }^{3}$ indican que la complejidad etiológica y manifestación de los trastornos por déficit de atención con hiperactividad en la infancia sigue sin comprenderse en su totalidad. Asimismo, estos autores señalan que podemos considerar dos grandes grupos de diferencias, neuropsicológicas o cognitivas, al comparar a los niños con TDAH con un grupo de control: en el funcionamiento ejecutivo y en la motivación.

Desde la psicología cognitiva y neuropsicología, habitualmente el trastorno por déficit de atención con hiperactividad es explicado como una disfunción ejecutiva o por déficit en las funciones ejecutivas ${ }^{4567}$. Muriel Lezak ${ }^{8}$ indica que dentro de las funciones ejecutivas se pueden diferenciar cuatro componentes: 1) volición; 2) planificación; 3) acción propositiva, con finalidad; y 4) rendimiento efectivo. Cada uno de estos componentes incluye un conjunto distintivo de comportamientos asociados a la actividad. Por otro lado, como indica Lezak (8) paradójicamente, la evaluación cognitiva tradicional, altamente estructurada, no es capaz de detectar déficit importantes en las funciones ejecutivas. Ella define la volición como la conducta intencional, e incluye la motivación y la conciencia de uno mismo, la conciencia del estado físico, la conciencia del entorno y contexto y la conciencia social. De este modo, se entiende que para que una intención se lleve a cabo y, finalmente se realice la actividad, es preciso que se inicie, se mantenga, se cambie una secuencia de comportamientos complejos de forma ordenada e integrada, pudiendo afectar a la motivación, conocimiento o la capacidad para realizar una actividad (8)

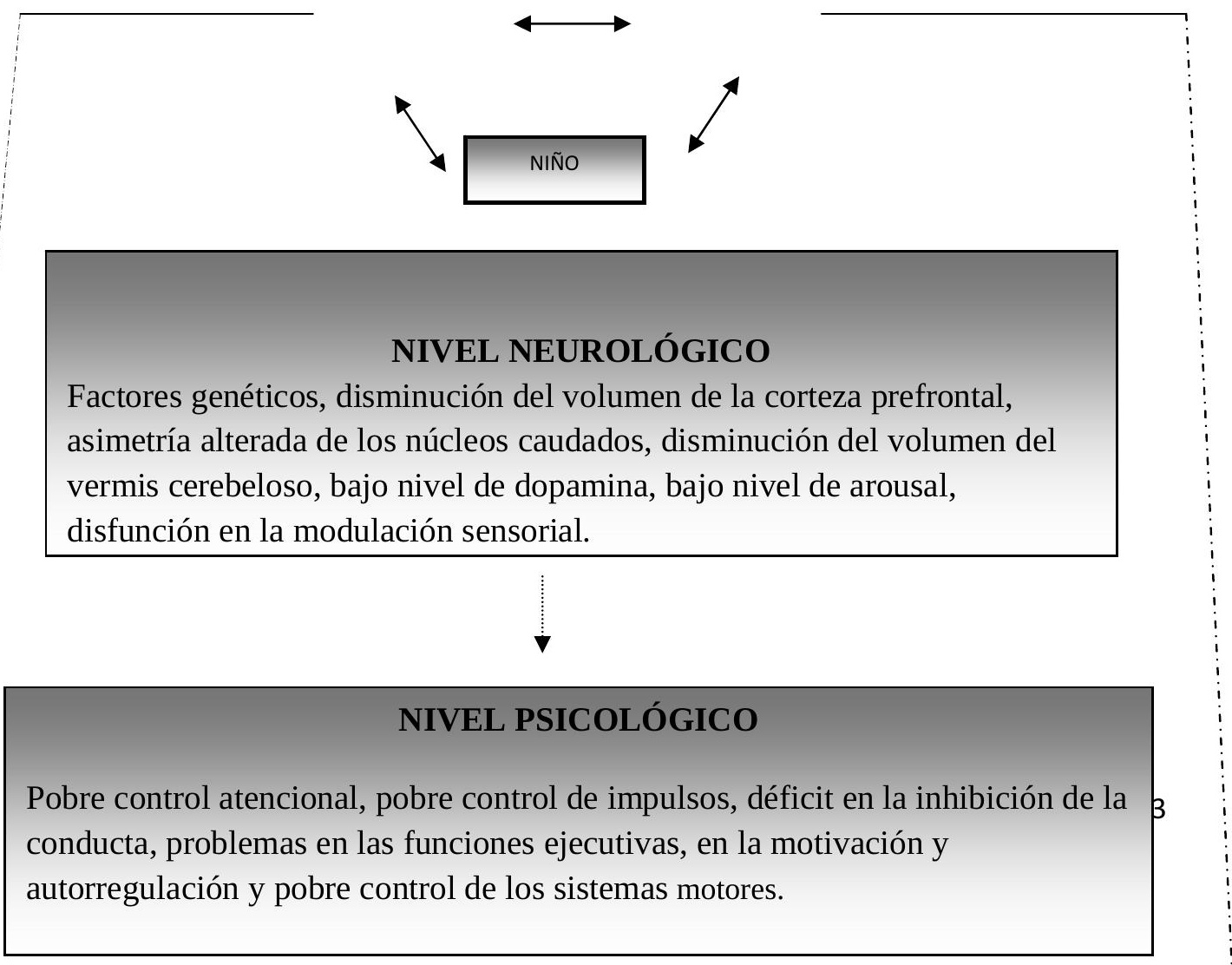




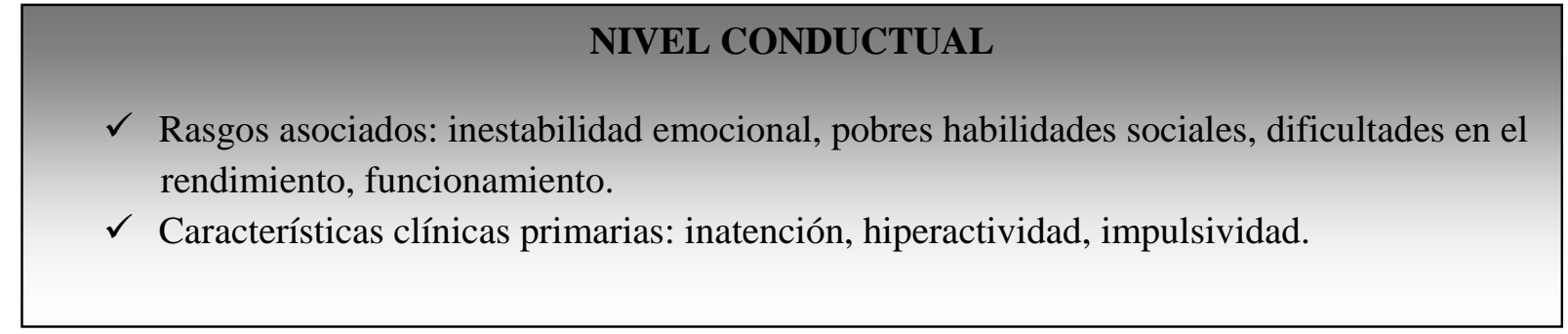

Figura 1. Modelo de TDAH como un trastorno adaptativo (2)

Por otro lado, uno de los modelos teóricos más conocidos sobre los trastornos por déficit de atención con hiperactividad, el modelo híbrido del TDAH $(5,6)$, explica que el déficit nuclear del trastorno es la autorregulación. Según el modelo híbrido la autorregulación conlleva los siguientes elementos: la conducta inhibitoria, las funciones ejecutivas y el sistema de control motor. De este modo, la conducta inhibitoria permite la demora de respuesta a un acontecimiento. Las funciones ejecutivas ponen en juego varios elementos: 1) la volición, planificación y la conducta intencional o dirigida a una meta; 2) la inhibición y resistencia a la distracción; 3) las acciones para solucionar problemas, para cumplir las demandas de la tarea; 4) el cambio flexible de acciones para lograr las demandas de la tarea; 5) mantener la persistencia para lograr una meta y tener conciencia a lo largo del tiempo; y, finalmente, 6) las funciones ejecutivas dirigen y guía la conducta productiva a través del sistema de control motor. Una vez que una meta ha sido elegida y se pone en acción por el sistema de control motor, es la conducta inhibitoria la que protege a estas conductas de ser interrumpidas por eventos distractores. Según Russell Barkley, los niños con TDAH presentaran un patrón conductual de inatención, hiperactividad e impulsividad debido a un déficit en la conducta inhibitoria. Desde una perspectiva cognitiva, de las teorías del procesamiento de la información, Sagvolden y Sergeant ${ }^{9}$ explican que la sobreactividad de los niños con TDAH no ocurre en todas las situaciones, es decir, depende de los contextos. Así por ejemplo, es más frecuente en la clase pero puede no estar presente en el juego y puede estar ausente en situaciones nuevas, incluso puede ocurrir que el nivel de actividad sea normal al comenzar una actividad pero se incremente a medida que transcurre la actividad. Para estos autores, el arousal es un elemento clave para entender estos trastornos.

Por otro lado, la mayoría de los estudios sobre motivación se centran en el estudio de los efectos de los refuerzos y los mecanismos biológicos relacionados con los mismos, especialmente en el papel de la dopamina y de la demora al refuerzo. ${ }^{10}$ Lo cierto es que, a pesar de considerarse que la motivación es un proceso alterado en este tipo de trastornos, la inmensa mayoría de los estudios se han centrado sobre el estudio de la 
motivación extrínseca, existiendo un mayor vacío de trabajos cuyo objeto de estudio sea la motivación intrínseca, la conducta intencional o volición.

Es bien sabido que el éxito de todo tratamiento está supeditado a los resultados obtenidos durante la evaluación. Cuanto más exhaustiva sea la evaluación más fácil será diseñar el tratamiento. En términos generales, podemos admitir que la terapia ocupacional pretende ayudar al individuo a realizar sus ocupaciones, dotándole de competencias, habilidades y estrategias que le permitan desempeñar sus tareas y roles en la vida diaria, modificando o adaptando las tareas, actividades o el entorno. Por tanto, una de las funciones esenciales del terapeuta ocupacional consiste en estimar las necesidades, dificultades y puntos fuertes del funcionamiento ocupacional en el contexto concreto de desempeño ocupacional.

Desde el Modelo de la Ocupación Humana (10), la volición o la conducta intencional en la infancia puede ser entendida como el conjunto de sentimientos, pensamientos, creencias que nos impulsan o conducen a realizar una determinada actividad, en función de las consideraciones acerca de uno mismo como actor en el mundo, lo que considera importante (valores), percibe como capacidad personal y efectividad (causación personal) y encuentra placentero (intereses). Este proceso sucede en distintos procesos a través de lo que el niño anticipa, elige, experimenta e interpreta lo que hace. Estos procesos permiten que la conducta intencional se desarrolle en tres etapas, de menor a mayor complejidad: exploración, competencia y logro. Se han llevado a cabo pocos estudios para analizar ¿qué sucede con la volición y motivación intrínseca de estos niños por determinadas ocupaciones socialmente relevantes?. Se ha explicado el desarrollo del autocontrol, a través del concepto de motivación de logro, que incluye procesos cognitivos que permiten al niño distinguir entre causación interna y causación externa. Así, si los resultados de sus actividades son consistentes a lo largo de los acontecimientos y son vistos como auto-producidos, entonces el niño desarrolla autocontrol.

Los resultados de la investigación realizada por Galejs ${ }^{11}$ muestra que la motivación de logro va aumentado a lo largo de los primeros años escolares. Pero es diferente en el caso de los niños y de las niñas. Así, en este estudio se ha observado que las niñas desarrollan antes conductas orientadas al logro. Según estos autores esto se debe a que los profesores tienen más interacciones e intercambio verbal con las niñas que con los niños. Del mismo modo, se observó que los niños con una percepción alta de control interno están más involucrados, entusiasmados y orientados a la tarea que los niños con una percepción de control baja. En conclusión, las percepciones de control de los niños preescolares sobre los resultados de los acontecimientos, predicen el desarrollo de la motivación de logro. Esta motivación parece estar centrada alrededor de la implicación y el interés, orientación verbal, y conductas orientadas a la tarea.

Farmer et al. ${ }^{12}$ indican que la motivación de logro es altamente dependiente del contexto. Así, estos autores reflejan que una persona puede estar motivada para realizar una actividad en un determinado entorno, pero no en otro. Gran parte de la investigación realizada sobre la motivación de logro hace referencia a la teoría del Aprendizaje Social de Bandura, de tal modo que se considera las experiencias ambientales sobre el aprendizaje como recíprocas y dinámicas y los procesos del aprendizaje cognitivo son estudiados en relación con la influencia de los factores ambientales.

Por otro lado, Vlachau y Farrel $^{13}$ hicieron un estudio con niños con y sin discapacidad para observar las diferencias en la motivación de logro. Estos autores apoyan su estudio 
en la premisa subyacente en los estudios de Piaget, entendiendo que el desarrollo del niño es muy dependiente de su esfuerzo inherente para la competencia. En este sentido, se entiende que el desarrollo de la competencia es dependiente de factores intrínsecos que impulsan al niño desde el nacimiento a una progresión en el compromiso en actividades exploratorias y sociales, incluyendo juego con objetos. Los factores intrínsecos constituyen una necesidad inherente para dominar el entorno, una necesidad de obtener placer desde la práctica de las destrezas emergentes, para explorar y tener control sobre el entorno, persistiendo en una conducta dirigida a una tarea.

Alessandri $^{14}$ estudió cómo se relacionaba la atención de los niños con TDAH en contextos de juego. Realizó un estudio con niños entre 4 y 5 años, con una muestra de 20 niños con TDAH y 20 controles. Durante 6 semanas grabaron interacciones sociales y de juego y situaciones de clase. El objetivo era analizar el nivel de atención, juego cognitivo y la participación social de estos niños. Los resultados de este estudio señalan que los niños con TDAH se involucran menos en el juego en general y más en juego sensoriomotor. Igualmente este estudio concluye que los niños son menos competentes con sus iguales y menos atentos y cooperativos durante las actividades de grupo.

Un instrumento útil para estudiar la volición en los niños es el Pediatric Volitional Questionnaire (PVQ) ${ }^{15} 1617{ }^{18}$. Aunque el nombre indica que es un cuestionario, en realidad son un conjunto de indicadores que se deben observar en distintas situaciones.

El objetivo de nuestro trabajo, como adelantábamos previamente, se centra en conocer cómo es la conducta intencional de una pequeña muestra de niños con TDAH y la utilidad del empleo del Pediatric Volitional Questionnaire (PVQ) en este ámbito.

Según los datos previos, la hipótesis para este estudio debería ser que dado que la atención sostenida es deficitaria en el grupo de niños del subtipo combinado (en adelante TDAH-C) pero no en el subtipo inatento (en adelante TDAH-I), los primeros también mostrarán más dificultades en la volición o conducta intencional que los niños TDAH-I, ya que su dificultad es en la atención selectiva (5). Los niños con TDAH (en general, los tres subgrupos) tienen más dificultades en la "Causalidad Personal” y precisan más feedback inmediato y positivo para construir una identidad ocupacional y lograr una adaptación ocupacional a su entorno. Los niños con TDAH muestran una baja volición, especialmente evidente en el nivel de competencia (probablemente debido a la baja causalidad personal). Ahora bien ¿cómo pueden modular los factores ambientales esto? En los niños del subtipo inatento ¿cómo es la conducta intencional de los niños con inatención (TDAH-I)?.¿Se ven afectados también los componentes afectivos de la volición: sentido de capacidad, de competencia personal?

La explicación de todo esto podría orientar a futuras intervenciones más holistas, en las que se contemple la necesidad de utilizar distintos tipos de estrategias en el tratamiento en distintos contextos: familiar y escolar, con el objetivo de mejorar la causalidad personal y analizar y usar aquellas actividades que permitan al niño ir avanzando en los distintos niveles volitivos y ocupacionales en la vida diaria, como actividades escolares, etc. 


\section{METODO}

\section{Muestra}

La muestra inicial estaba formada por 25 niños con el diagnóstico de Trastorno por Deficit de Atención con Hiperactividad según los criterios diagnósticos del DSM-IV-R. De ellos, 4 niños no cumplían los criterios de nuestro estudio por mostrar tres de ellos retraso mental moderado y un niño mostraba una conducta disruptiva, pero no problemas de atención. Finalmente, la muestra quedó formada por 21 niños, tal y como muestra el Gráfico 1. La edad de la muestra oscila entre los 7 años y los 11 años $(X=$ $8,5 ; S D=1,1)$. De ellos, cuatro niños mostraban dominancia manual izquierda y diecisiete niños dominancia manual derecha según el cuestionario de lateralidad de Edimburgo.

\section{Gráfico 1}

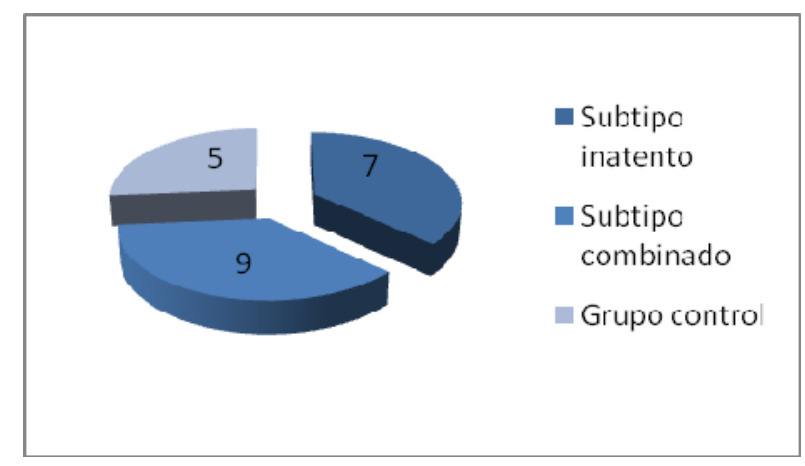

\section{Instrumentos}

Durante el proceso de evaluación se emplearon instrumentos para utilizar en distintos contextos. En la evaluación, se utilizó el cuestionario de lateralidad de Edimburgo y la escala de inteligencia de Wechsler para niños revisada. Para evaluar la conducta intencional o volición empleamos el Pediatric Volitional Questionnaire (PVQ) versión 2.0. (16). Este instrumento está desarrollado bajo las premisas del Modelo de la Ocupación Humana. Aunque este instrumento puede utilizarse con niños entre los 2 y 7 años y existe una versión para adultos (Volitional Questionnaire), elegimos este cuestionario por ser más adecuados los ítems y poder ser comparados en futuros estudios con niños menores. Los ítems son los siguientes:

1. Explora la novedad

2. Inicia acciones

3. Se encuentra dirigido a la tarea

4. Muestra preferencias

5. Intenta cosas nuevas

6. Permanece involucrado

7. Expresa placer por el logro

8. Intenta resolver problemas

9. Intenta producir efectos

10. Practica habilidades

11. Busca desafíos

12. Organiza / modifica el medio ambiente 
13. Realiza la actividad hasta terminarla

14. Utiliza la imaginación / simbolismo

Cada uno de los ítems debe valorarse según el manual de aplicación del PVQ, pudiéndose calificar como: 1 = pasivo; $2=$ dudoso; 3= involucrado; 4= espontáneo.

Las ventajas que ofrece este instrumento frente a otro tipo de evaluación sobre la volición, es que utiliza una metodología observacional, permite tener en cuenta el entorno del niño y cómo influye éste en el desempeño ocupacional, aunque requiere que la observación se realice en al menos dos contextos y esto supone un aumento de tiempo en la evaluación. Por otro lado, los profesores cumplimentaron la Evaluación del Déficit de Atención e Hiperactividad (Farré y Narbona), que es una adaptación de la escala de Conners. Además de la información obtenida directamente con las distintas pruebas realizadas por el niño, se realizó una entrevista a los padres para obtener información sobre el comportamiento y actividades en el hogar, y se les pidió que cumplimentasen el CBCL (Child Behavior Checklist).

\section{Procedimiento}

El estudio fue aprobado por el comité ético del Hospital Ntra. Sra. Del Prado (Talavera de la Reina). Todos los niños del grupo experimental fueron derivados por el Servicio de Salud Mental Infanto- Juvenil del Hospital de Talavera de la Reina. En una entrevista inicial con los padres se les explicó el objetivo del estudio y se obtuvo el consentimiento informado de los padres para participar en el mismo. Un terapeuta ocupacional entrenado en el uso del PVQ realizó las observaciones en el hogar durante una semana en el horario de tarde, entre las 17- 20 horas, observando dos tipos de actividades: actividades escolares, tareas escolares y actividades de la vida diaria. Además, esta misma información y las puntuaciones fueron cotejadas con los padres de los niños. Se optó por hacer una observación durante una semana, para evitar la reactividad ante la observación y las puntuaciones se realizaban en el $4^{\circ}$ ó $5^{\circ}$ día de observación. La terapeuta u observador era presentada como una amiga de la mamá que venía a ayudar durante esa semana.

\section{Análisis de Datos}

Los datos han sido analizados utilizando el paquete estadístico SPSS versión 17.0. Las prueba estadística empleada, tras la realización de la tabla de contingencia, dado el tamaño de la muestra y el tipo de variables, ha sido la Chi-cuadrado, con un nivel de significación $\mathrm{p}<0.05$.

\section{RESULTADOS}

\section{Comparación por grupos diagnósticos}

Los resultados al comparar los tres grupos muestran que existen diferencias significativas en un único ítem al observar las actividades de la vida diaria: " utiliza la imaginación" $(p=, 006)$. En cambio, de los resultados de las tareas escolares se desprende que hay diferencias significativas en los siguientes ítems: "iniciar acciones en las tareas escolares” ( $p=, 018)$, “permanece involucrado en tareas escolares” $(p=, 004)$, "muestra 
preferencias en tareas escolares" ( $p=, 016)$, "practica habilidades" $(p=, 001)$, "busca desafíos" ( $p=, 005)$, “termina las tareas escolares" $(p=, 031)$. Son marginalmente significativos los ítem: “muestra curiosidad en tareas escolares" $(p=, 087)$ y "modifica el entorno" $(p=, 075)$. Estas diferencias siempre se han debido a una puntuación inferior en los niños del subtipo combinado respecto al grupo control y al grupo de niños del subtipo inatento.

En la Tabla 1 se resumen los resultados de la prueba Chi-cuadrado en los diferentes ítems del PVQ.

Tabla 1. Resultados en la prueba de Chi-cuadrado en los ítems del PVQ

\begin{tabular}{|c|c|c|}
\hline & $\begin{array}{l}\text { Diferencias significativas } \\
(\mathrm{p}<0.05)\end{array}$ & $\begin{array}{l}\text { Sin diferencias significativas } \\
(\mathrm{P}>0.05)\end{array}$ \\
\hline $\begin{array}{l}\text { Conducta intencional en } \\
\text { actividades de la vida diaria } \\
\text { en el hogar }\end{array}$ & - Utiliza la imaginación & $\begin{array}{ll}- & \text { Expresar satisfacción por sus } \\
- & \text { logros } \\
- & \text { Practica habilidades } \\
- & \text { Iniciar acciones } \\
- & \text { Mostrar curiosidad/explorar } \\
- & \text { Intentar cosas nuevas } \\
- & \text { Permanecer involucrado } \\
- & \text { Muestra preferencias } \\
- & \text { Intentar producir efectos } \\
- & \text { Buscar desafíos } \\
- & \text { Realiza la actividad hasta } \\
- & \text { completarla } \\
- & \text { Se encuentra dirigido a la tarea } \\
- & \text { Organiza/modifica el entorno } \\
\text { Intentar resolver problemas }\end{array}$ \\
\hline $\begin{array}{l}\text { Conducta intencional en } \\
\text { actividades escolares en el } \\
\text { hogar }\end{array}$ & 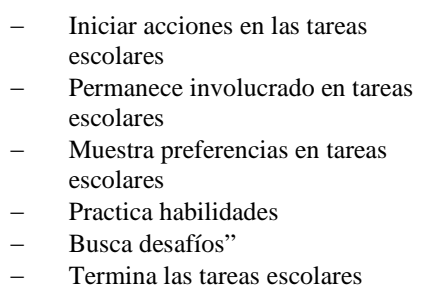 & $\begin{array}{ll}- & \text { Explora la novedad } \\
- & \text { Se encuentra dirigida a la tarea } \\
- & \text { Intentar cosas nuevas } \\
- & \text { Intenta resolver problemas } \\
- & \text { Intenta producir efectos } \\
- & \text { Organiza/modifica el ambiente } \\
- & \text { Utiliza la imaginación }\end{array}$ \\
\hline
\end{tabular}

El grado de dificultad de los ítems para las dos ocupaciones estudiadas se muestran ordenadas de menor a mayor en la Tabla 2 y Tabla 3.

Tabla 2. Volición en Actividades de la Vida Diaria en niños entre 7 y 11 años.

\begin{tabular}{|c|c|c|c|c|c|c|c|}
\hline \multirow[b]{2}{*}{ Item } & \multirow[b]{2}{*}{ Media } & \multicolumn{5}{|c|}{ Categoría de la Escala } & \multirow[b]{2}{*}{$\begin{array}{l}\text { Valores } \\
\text { Perdidos } \\
\end{array}$} \\
\hline & & $\begin{array}{l}\text { Desviación } \\
\text { Típica } \\
\end{array}$ & Pasivo & Dudoso & Involucrado & Espontáneamente & \\
\hline Expresar satisfacción por su logros & 3,8 &, 511 & 0 & 1 & 2 & 18 & 0 \\
\hline Practica habilidades & 3,48 & ,97 & 1 & 4 & 1 & 15 & 0 \\
\hline Iniciar acciones & 3,47 & ,67 & 0 & 2 & 7 & 12 & 0 \\
\hline Mostrar curiosidad/explorar & 3,38 & ,74 & 0 & 3 & 7 & 11 & 0 \\
\hline Intentar cosas nuevas & 3,23 & 1,09 & 2 & 4 & 2 & 13 & 0 \\
\hline Permanecer involucrado & 3,23 & ,83 & 0 & 5 & 6 & 10 & 0 \\
\hline Muestra preferencias & 3,19 & ,98 & 0 & 8 & 1 & 12 & 0 \\
\hline Intentar producir efectos & 3,19 & ,99 & 1 & 8 & 2 & 10 & 0 \\
\hline Buscar desafíos & 3,00 & 1,09 & 2 & 6 & 3 & 10 & 0 \\
\hline Realiza la actividad hasta completarla & 2,80 & 87 & 1 & 7 & 8 & 5 & 0 \\
\hline Se encuentra dirigido a la tarea & 2,71 & ,90 & 6 & 3 & 5 & 7 & 0 \\
\hline Organiza/modifica el entorno & 2,71 & ,90 & 1 & 9 & 6 & 5 & 0 \\
\hline Intentar resolver problemas & 2,66 & ,79 & 1 & 8 & 9 & 3 & 0 \\
\hline
\end{tabular}




\begin{tabular}{llllllll}
\hline Utiliza la imaginación & 2,61 & 1,24 & 6 & 3 & 5 & 7 & 0 \\
& & & & & & & \\
\end{tabular}

En ambos casos, se observa que los ítems más difíciles, los que tienen una media menor, son los que corresponden a la etapa de logro de la conducta volitiva o intencional.

Tabla 3. Volición en Actividades Escolares en niños entre 7 y 11 años.

\begin{tabular}{|c|c|c|c|c|c|c|c|}
\hline \multirow[b]{2}{*}{ Item } & \multirow[b]{2}{*}{ Media } & \multicolumn{5}{|c|}{ Categoría de la Escala } & \multirow[b]{2}{*}{$\begin{array}{l}\text { Valores } \\
\text { Perdidos } \\
\end{array}$} \\
\hline & & $\begin{array}{l}\text { Desviación } \\
\text { Típica } \\
\end{array}$ & Pasivo & Dudoso & Involucrado & Espontáneamente & \\
\hline Intentar cosas nuevas & 2,76 & ,99 & 2 & 7 & 6 & 6 & 0 \\
\hline Practica habilidades & 2,71 & ,90 & 1 & 9 & 6 & 5 & 0 \\
\hline Iniciar acciones & 2,66 & ,79 & 0 & 11 & 6 & 4 & 0 \\
\hline Intentar resolver problemas & 2,47 & ,98 & 4 & 8 & 6 & 3 & 0 \\
\hline Intentar producir efectos & 2,47 & ,98 & 3 & 8 & 6 & 4 & 0 \\
\hline Permanecer involucrado & 2,38 & ,80 & 1 & 14 & 3 & 3 & 0 \\
\hline Realiza la actividad hasta completarla & 2,38 & ,80 & 3 & 12 & 3 & 3 & 0 \\
\hline Organiza/modifica el entorno & 2,33 & 91 & 3 & 11 & 4 & 3 & 0 \\
\hline Buscar desafíos & 2,23 & ,94 & 4 & 11 & 3 & 3 & 0 \\
\hline
\end{tabular}

Por otro lado, los resultados de cada grupo también muestran que los ítems de logro son los más complejos para los tres grupos, (niños con predominio inatento, los pertenecientes al grupo combinado y del grupo control). Sin embargo, también se observa que los niños del subtipo combinado muestran menos espontáneamente la conducta intencional, obteniendo puntuaciones inferiores en el desarrollo del sentido de capacidad y competencia, así como en el desarrollo del logro.

Tabla 4. Conducta intencional en Actividades de la Vida Diaria y Tareas Escolares en niños entre 7 y 11 años.

\begin{tabular}{lllllll}
\hline & Grupo TDAH-C & \multicolumn{2}{l}{ GRUPO TDAH-I } & \multicolumn{2}{c}{ CONTROL } \\
\hline Item & Media & $\begin{array}{l}\text { Desviación } \\
\text { Típica }\end{array}$ & Media & $\begin{array}{l}\text { Desviación } \\
\text { Típica }\end{array}$ & Media & $\begin{array}{l}\text { Desviación } \\
\text { Típica }\end{array}$ \\
\hline Actividades de la Vida Diaria & & & & & & \\
Explora la novedad & 3,33 &, 86 & 3,28 &, 75 & 3,75 &, 50 \\
Inicia acciones & 3,33 &, 86 & 3,42 &, 53 & 3,75 &, 50 \\
Se encuentra dirigido a la tarea & 1,55 &, 88 & 3,14 &, 89 & 4 &, 00 \\
Muestra preferencias & 2,88 & 1,05 & 3,28 &, 95 & 4 &, 00 \\
Intenta cosas nuevas & 2,88 & 1,16 & 3,71 &, 75 & 4 &, 00 \\
Permanece involucrado & 3,00 &, 88 & 3,28 &, 75 & 4 &, 00 \\
Expresa placer por el logro & 3,88 &, 76 & 4 &, 00 & 4 &, 00 \\
Intenta resolver problemas & 2,22 &, 66 & 2,71 &, 75 & 3,5 &, 57 \\
Intenta producir efectos & 2,88 & 1,16 & 3,71 &, 75 & 4 &, 00 \\
Practica habilidades & 2,88 & 1,16 & 3,71 &, 95 & 4 &, 00 \\
Busca desafíos & 2,44 & 1,13 & 3,14 & 1,06 & 4 &, 00 \\
Organiza / modifica el medio ambiente & 2,11 &, 60 & 2,85 &, 89 & 3,75 &, 50 \\
Realiza la actividad hasta terminarla & 2,33 & 1,16 & 3,14 &, 69 & 3,50 &, 57 \\
Utiliza la imaginación / simbolismo & 2,44 & 1,13 & 3,14 & 1,06 & 4 &, 00 \\
Tareas escolares & & & & & \\
Explora la novedad & 1,88 &, 78 & 2,71 &, 95 & 3,50 &, 57 \\
\hline
\end{tabular}




\begin{tabular}{lllllll}
\hline Inicia acciones & 2,44 &, 52 & 2,42 &, 78 & 3,75 &, 50 \\
Se encuentra dirigido a la tarea & 1,66 &, 70 & 2,28 & 1,38 & 3,50 &, 57 \\
Muestra preferencias & 2,11 & 1,36 & 2,57 &, 78 & 4 &, 00 \\
Intenta cosas nuevas & 2,33 & 1,11 & 3 &, 81 & 3,50 &, 57 \\
Permanece involucrado & 1,88 &, 33 & 2,57 &, 97 & 3,25 &, 50 \\
Expresa placer por el logro & 3,77 &, 44 & 4 &, 00 & 4 &, 00 \\
Intenta resolver problemas & 2,22 &, 97 & 2,28 &, 95 & 3,50 &, 57 \\
Intenta producir efectos & 2,33 &, 70 & 2,42 &, 78 & 4 &, 00 \\
Practica habilidades & 2,33 &, 70 & 2,42 &, 78 & 4 &, 00 \\
Busca desafíos & 1,66 &, 70 & 2,14 &, 69 & 3,75 &, 50 \\
Organiza / modifica el medio ambiente & 2,00 &, 70 & 2,14 &, 89 & 3,50 &, 57 \\
Realiza la actividad hasta terminarla & 1,88 &, 33 & 2,42 &, 95 & 3,50 &, 57 \\
Utiliza la imaginación / simbolismo & 1,66 &, 50 & 2,14 & 1,06 & 4 &, 00
\end{tabular}

En las Figuras 2 y 3 se puede observar las diferencias entre las puntuaciones en los distintos ítems en los tres grupos estudiados.

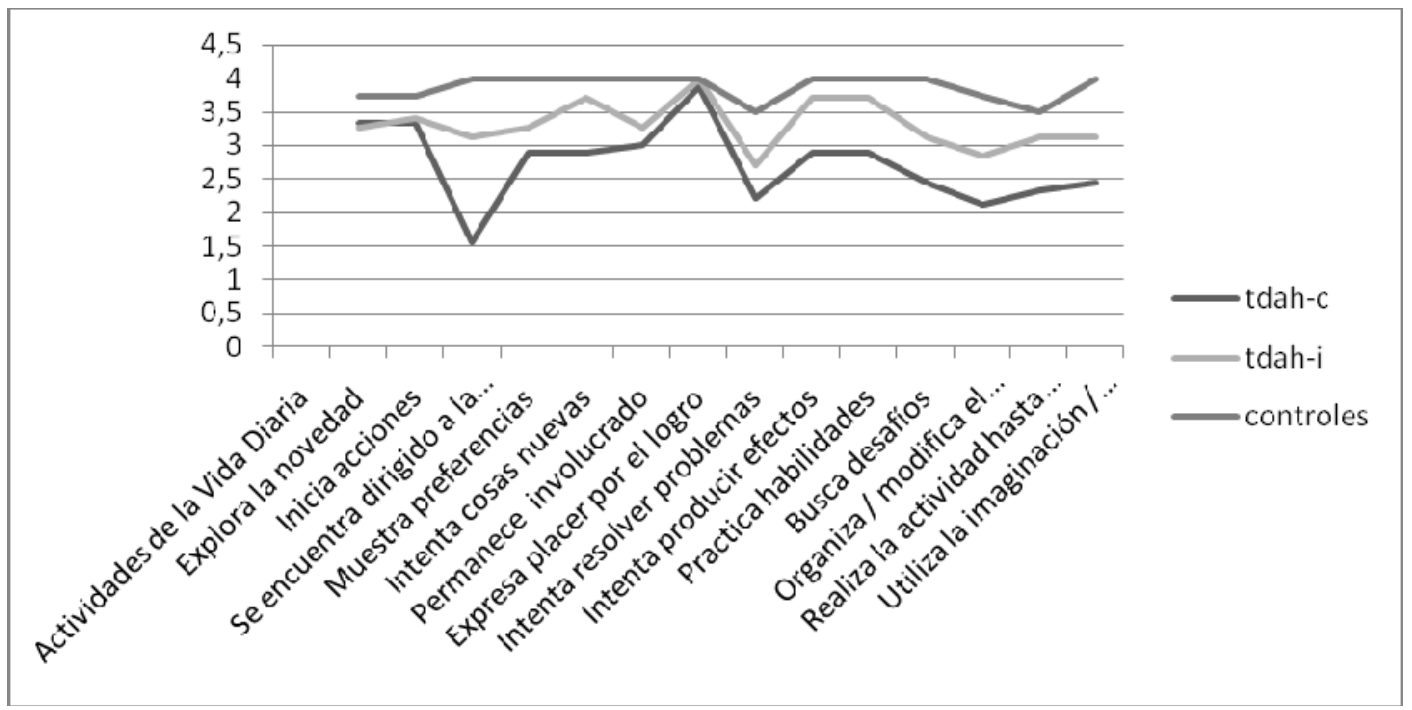

Figura 2. Comparación de puntuaciones medias en la conducta intencional en Actividades de la Vida Diaria

Como muestran las figuras, en ambas ocupaciones el ítem más sencillo para todos los grupos estudiados es el de "expresar satisfacción o placer por el logro". Igualmente, se observa que para el grupo de niños de TDAH-C los ítems más difíciles son: encontrarse dirigido a la tarea, permanecer involucrado, buscar desafíos, organizar o modificar el medio ambiente y utilizar la imaginación en las tareas escolares. 


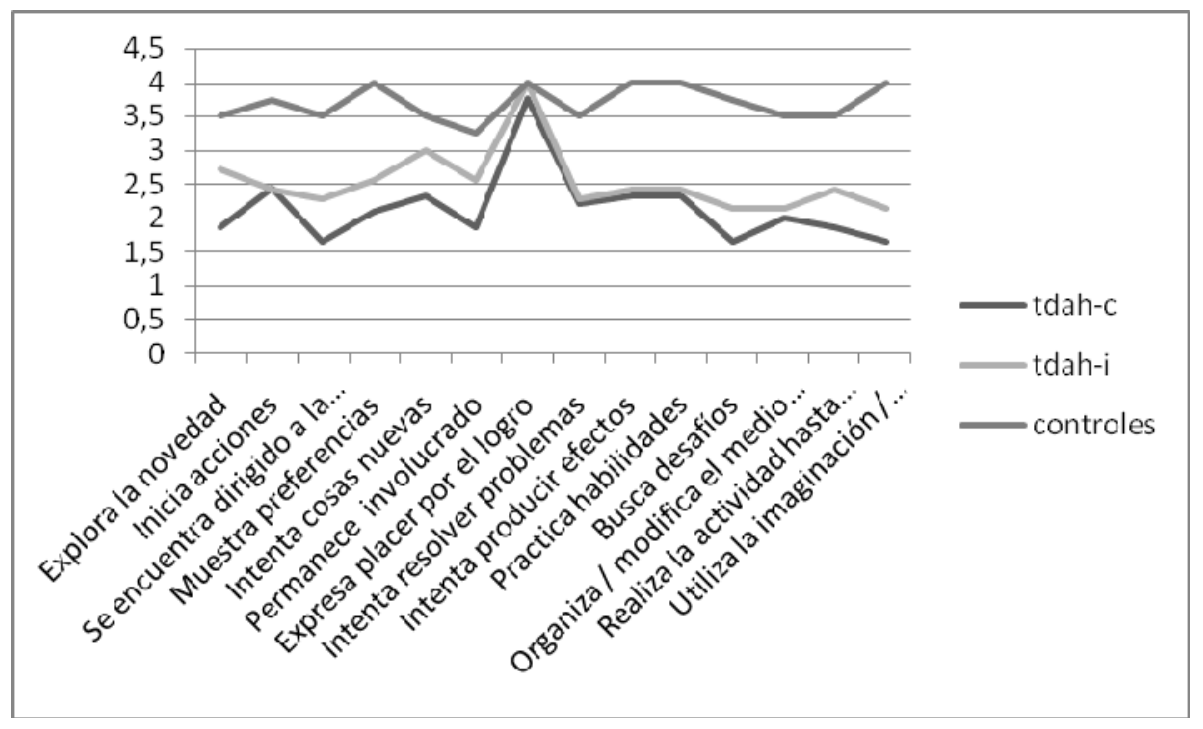

Figura 3. Comparación de puntuaciones medias en la conducta intencional en Tareas Escolares

\section{DISCUSIÓN Y CONCLUSIONES}

Los resultados anteriores nos permiten verificar la utilidad del instrumento PVQ para la evaluación de la conducta intencional de los niños con TDAH desde un modelo teórico propio de la Terapia Ocupacional. Además, permite conocer detalladamente qué etapas del desarrollo de la conducta intencional o volición están afectadas y permite establecer objetivos de tratamiento más específicos y dirigidos a la situación de cada niño.

Los resultados anteriores están en parte de acuerdo con los encontrados por Ruskin et al. ${ }^{19}$, quien ha señalado que los niños con discapacidad muestran mayores dificultades para persistir en la tarea, pero en cambio son diferentes en cuanto a los resultados referentes a que los niños con dificultades en el aprendizaje tienen muestran menos afecto positivo contingente sobre la solución de la tarea. Nuestros hallazgos indican que los niños con TDAH, independientemente del subtipo clínico no manifiestan dificultades en expresar placer por el logro.

Por otro lado, en un estudio realizado por Dunn y Shapiro ${ }^{20}$ señalan que el $85 \%$ de los niños con TDAH (a diferencia del grupo control sólo un $40 \%$ ) eligen tareas que les permiten manifestar sus destrezas a otros más que el hecho de aprender nuevas habilidades. Nuestros hallazgos sugieren que los niños con TDAH buscan menos desafíos y practican menos habilidades que los niños del grupo control. Estas diferencias pueden ser debidas, primero al reducido tamaño de la muestra, en segundo lugar, a las influencias del entorno, del contexto y a la propia actividad u ocupación que sea considerada al estudiar la conducta volitiva, de acuerdo con lo planteado en el modelo de Jensen et al. (2). Y, por último, a que en nuestro estudio hemos diferenciado los dos subtipos principales para poder compararlos. Así, por ejemplo, si tomamos los resultados de los niños con predominio inatento se observa que practican más 
habilidades que el subtipo combinado, tanto en las actividades de la vida diaria como en las actividades escolares.

De acuerdo con los resultados obtenidos por Geist, Kielhofner, Lai (18) y por Basu (16,17) encontramos que los ítems más fáciles o sencillos son los que tienen que ver con la primera etapa del desarrollo de la conducta intencional o volición: "explora la novedad”, “inicia acciones”, "se encuentra dirigido a la tarea”, “muestra preferencias”, "intenta cosas nuevas" y "permanece involucrado". Todas las conductas en este nivel implican la interacción con el entorno con el propósito de descubrir la información en el mismo. Sin embargo, a este primer grupo tendríamos que añadirle el ítem "expresar satisfacción por el logro de la actividad”, que según el estudio previamente citado correspondería a la segunda etapa del desarrollo de la volición, relacionado con el sentido de eficacia personal y de la capacidad. Nuestros resultados nos pueden sugerir dos hechos: 1) que los niños en estas edades hacen una buena valoración de su propia habilidad; o 2) que se conforman rápidamente con los resultados, independientemente de que sean positivos. Esta última hipótesis es acorde con los planteamientos de otros autores como $\mathrm{Hoza}^{21}$ y Ohan ${ }^{22}$ sobre la protección de la autoestima. De tal manera que para proteger su propia estima se proporcionan un feedback positivo a pesar de tener conocimiento de sus errores o dificultades en la ejecución de la actividad.

También, de acuerdo con los resultados de Geist, Kielhofner, Lai (18), en los grupos de niños con TDAH el ítem "utilizar la imaginación/ simbolismo" supone una mayor dificultad porque requiere mayor cambio, ya que incluye la creencia en la habilidad para atribuir el propio significado a los objetos y actos simbólicos o escenarios imaginados.

Además, el estudio realizado por Basu (16,17) señala que los ítems practicar habilidades, intentar cosas nuevas, permanecer ocupado requieren un mayor sentido de competencia y capacidad para intentar mejorar las propias habilidades a través de la práctica, intentar una actividad nueva, continuar para seguir involucrado en una tarea y lograr realizar una tarea para terminar lo intentado. Nuestros resultados muestran que en el caso de las tareas escolares estos ítems son más difíciles para los niños con TDAH. Igualmente, esto ocurre en la etapa de logro, donde se intenta lograr un resultado con un estándar de éxito y responder a las expectativas del rendimiento.

Estos resultados preliminares apoyan la hipótesis del estudio respecto a la mayor dificultad para el desarrollo de la conducta intencional en la etapa de competencia y en consecuencia también de logro, de tal modo que presentan una baja causalidad personal, que queda reflejada en no practicar habilidades, no involucrarse con las actividades, no intentar resolver problemas, no buscar desafíos y no intentar (proponer) finalizar las actividades, afectando de forma dinámica al desarrollo de la atención sostenida a lo largo de la infancia y por tanto su rendimiento en diversas actividades cognitivas. En la Figura 4 presentamos de forma gráfica cómo se ve afectada la conducta intencional según los datos previos. Los resultados sugieren que un menor sentido de competencia o de capacidad puede afectar no sólo al rendimiento en distintas ocupaciones, sino también al desarrollo de la identidad ocupacional, adopción de roles y responsabilidades propias para la edad, así como afectar a la adaptación ocupacional, dadas las diferencias entre las demandas del contexto o actividad (ocupación) y el sentido de competencia y capacidad personal. En su conjunto, estos resultados preliminares sugieren que los tratamientos de terapia ocupacional con niños con TDAH (especialmente del subtipo combinado) deberían fomentar el desarrollo de un sentido positivo de competencia y de identidad ocupacional, graduando las actividades y el dominio de las mismas. De este modo, se puede ir permitiendo que el niño desarrolle progresivamente mayor 
autocontrol, sentido de competencia, de capacidad personal, y estar centrado mayoritariamente sobre el feedback externo. Esto es especialmente importante tenerlo en cuenta no sólo en el ámbito familiar, sino también en el escolar, donde el impacto de sus esfuerzos y el rendimientos en estas actividades son cruciales para el desarrollo del sentido de competencia personal en estas edades del desarrollo, por lo que los programas de intervención deberían considerar muy especialmente la intervención en el ámbito escolar y con los grupos de iguales, con los que la comparación es frecuente y relevante para desarrollo del sentido de competencia ocupacional, el desempeño de roles, el desarrollo de la identidad ocupacional y finalmente lograr la adaptación ocupacional a los contextos de desempeño de cada niño.

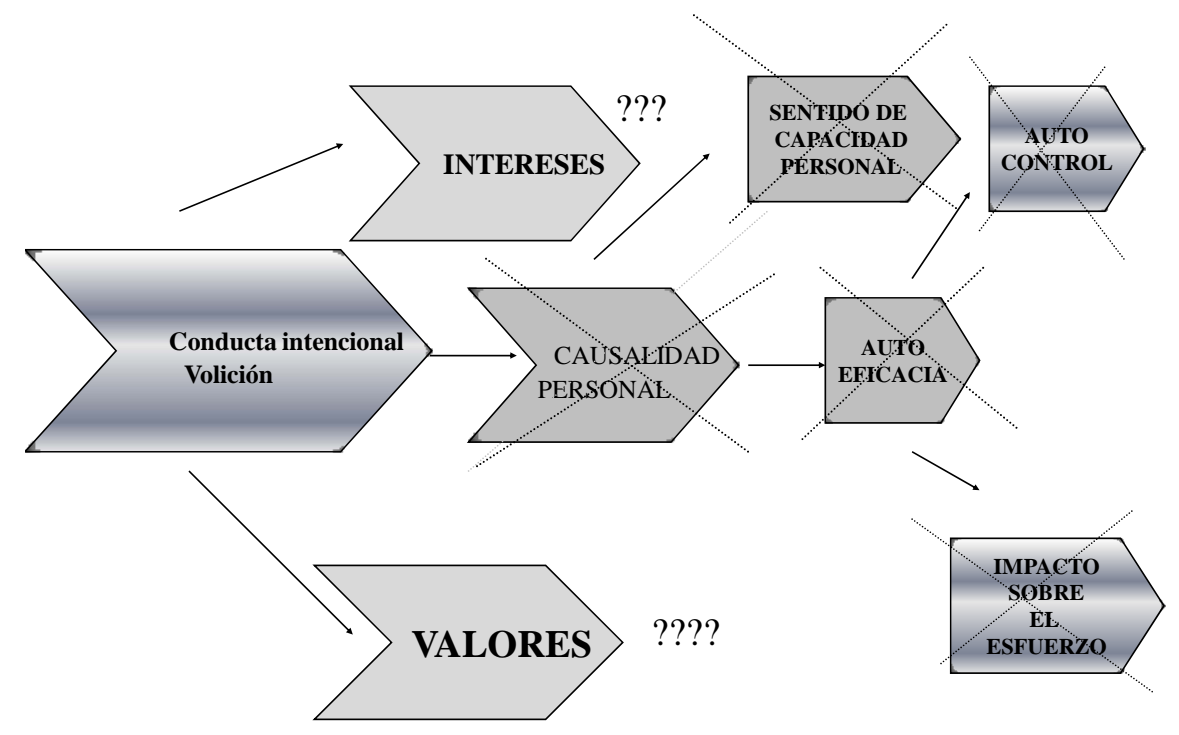

Figura 4. Elementos implicados en el desarrollo de la conducta intencional en los niños con TDAH

En trabajos posteriores sería interesante estudiar también cómo influyen los intereses, los valores y los procesos de atribución de competencia personal en el desarrollo de la conducta intencional. Por otro lado, hay que tener en cuenta que las conclusiones que se derivan del estudio que aquí presentamos son preliminares y es conveniente ampliar la muestra del estudio. 


\section{AGRADECIMIENTOS}

Este trabajo ha sido realizado gracias al apoyo de la Consejería de Salud de la Junta de Comunidades de Castilla - La Mancha, la Universidad de Castilla-La Mancha, la Unidad de Psiquiatría Infanto-Juvenil del $\mathrm{H}^{\mathrm{a}}$ Ntra. Sra. Del Prado de Talavera de la Reina. Queremos agradecer la participación de los niños del Colegio Ntra. Sra. De las Nieves de Madrid, el Colegio Hernán Cortés de Talavera de la Reina y el Colegio Pablo Iglesias de Talavera de la Reina.

\section{REFERENCIAS BIBLIOGRÁFICAS}

1 CHU, S. Attention deficit hyperactivity disorder (ADHD) part one: a review of the literature. International Journal of Therapy and Rehabilitation, 2003; 10(5), 218-227.

2 JENSEN, PS., MRAZEK D, KNAPP PK, STEINBERG L, PFEFFER C, SCHOWALTER J, SHAPIRO T. Evolution and revolution in child psychiatry: ADHD as a disorder of adaptation. Journal of American Academy of Child and Adolescent Psychiatry. 1997; 36(12):1672-1679

3 TRIPP, G. Y WICKENS, JR.Neurobiology of ADHD. Neuropharmacology, 2009.

${ }^{4}$ DIMOSKA A, JOHNSTONE SJ, BARRY RJ, CLARKE AR. Inhibitory motor control in children with attention/deficit/Hyperactivity disorder: event-related potentials in the stop-signal paradigm. Biol Psychiatry 2003; 54:1345-1354.

${ }^{5}$ OZONOFF S, JENSEN J. Specific Executive Function Profiles in Three Neurodevelopmental Disorders. J Autism Dev Disord 2001; 29(2):171-177.

${ }^{6}$ BARKLEY, R.A., Behavioral Inhibition, Sustained Attention and Executive Functions: Constructing a Unifying Theory of ADHD. Psychological Bulletin, 1997. 121(1): p. 65-94.

${ }^{7}$ BARKLEY, R. A. Issues in the diagnosis of attention-deficit/hyperactivity disorder in children. Brain and Development, 2003; 25, 77-83.

8 MURIEL D. L., DIANE B. H., DAVID D. L., HANNAY, J. \& FISHER J.L. Neuropsychological Assessment. 2004, New York: Oxford University Press

${ }^{9}$ SAGVOLDEN T. \& SERGEANT, J.A. Attention deficit/hyperactivity disorder from brain dysfunctions to behavior. Behavioural Brain Research,1998; 9, 1-10.

${ }^{10}$ MCINERNEY, R. \& KERNS, K. Time reproduction in children with ADHD: motivation matters. Child Neuropsychology, 2003, 9(2), 91-108. 
${ }^{11}$ KIELHOFNER, G. Teapia ocupacional. Modelo de la Ocupación Humana. Teoría y Aplicación, 2008, Buenos Aires: Editoria Médica Panamericana

${ }^{12}$ FARMER, H.S., VISPOEL, W. \& MAEHR, M.L. Achievement Contexts: Effect on achievement values and causal atributions. Journal of Educational Research, 1991, 85 (1): 26-38.

13 VLANCHOU, M. \& FARREL, P. Object Mastery Motivation in Pre-school Children with and without Disabilities. Educational Psychology, 2000, 20(2), 167176.

14 ALESSANDRI, S. Attention, play and social behavior in ADHD preschoolers. Journal of Abnormal Child Psychology, 1992, 20 (3), 289-302.

${ }^{15}$ ANDERSEN, S.,. KIELHOFNER, G. \&. LAI, J.S. An examination of the Measurement properties Pediatric Volitional Questionnaire, in Department of Occupational Therapy. 1998, University of Illinois at Chicago: Chicago. p. 30.

${ }^{16}$ BASU, S. The Peatric Volitional Questionnaire. The Model of Human Occupation 2002, Clearinghouse. Department of Occupational Therapy. University of Illonois at Chicago.

${ }^{17}$ BASU, S. The study of validity of Pediatric Volitional Questionnaire, 2003, Department of Occupational Therapy. University of Illinois at Chicago.

${ }^{18}$ GEIST, R., KIELHOFNER, G., BASU, S., \& KAFKES, A. The Pediatric Volitional Questionnaire (version 2.0.), 2002. Chicago: University of Illinois at Chicago.

19 RUSKIN EM, KASARI C, MUNDY P, SIGMAN M. Attention to people and toys during social and object mastery in children with Down syndrome. American Journal of Mental Retardation, 1994 99(1):103-11.

${ }^{20}$ DUNN, W. \& BENNET, D. Patterns of Sensory Processing in Children with Attention Deficit Hyperactivity Disorder. The Occupational Therapy Journal of Research, 2002, 22(1), 4-15.

${ }^{21}$ HOZA, B. PELMA, W.E. DOBBS, J. SARNI, J.; PILLOW, D.R. Do boys with attention-deficit /hyperactivity disorder have positive illusory self-concepts? Journal of Abnormal Psychology, 2002, 11 (2): 268-278.

${ }^{22}$ OHAN, J. \& JOHNSTON, C. Are the performance overstimates given by boys with ADHD self-protective?. Journal Clinical Child and Adolescent Psychology, 2002, 31 (2), 230-241. 